\title{
EVALUACIÓN DEL IMPACTO EN SEGURIDAD ALIMENTARIA DEL PROYECTO ESCUELAS DE CAMPO (ECAs), DIRIGIDO A PEQUEÑAS FAMILIAS CAMPESINAS DE LAS COMUNIDADES EL TERRERO Y EL MAMEY DEL MUNICIPIO DE SAN JOSÉ DE CUSMAPA, MADRIZ
}

\author{
Elgin Vivas Viachica1, Johanna del Carmen Herrera Ampie, Ahmed Avilés Sequeira
}

${ }^{1}$ PhD. Facultad de Desarrollo Rural, Universidad Nacional Agraria, apartado 453- e mail: elgin.vivas@una.edu.ni

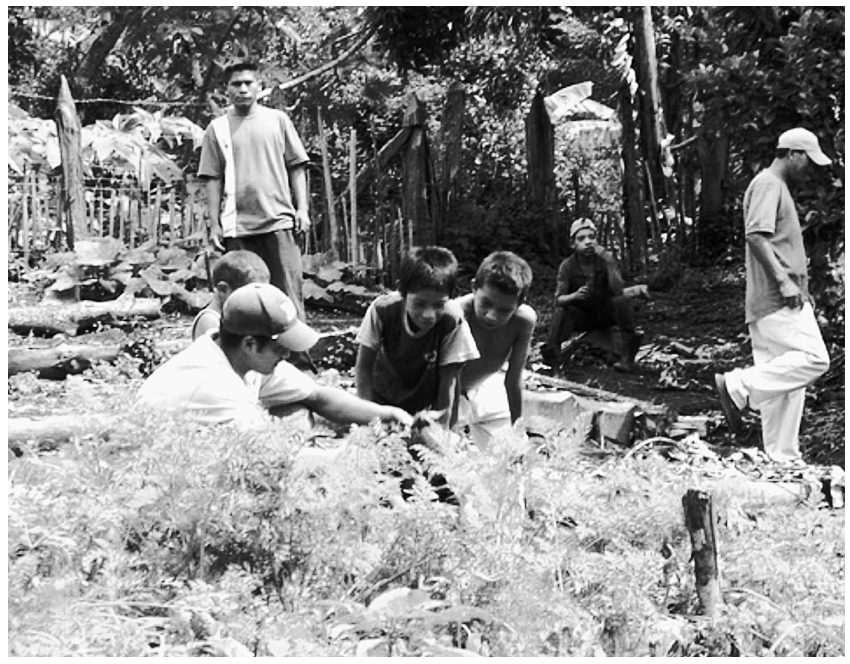

\section{RESUMEN}

La realidad imperante del municipio de San José de Cusmapa, Madriz, plantea la necesidad de ejecutar proyectos que aporten a la Seguridad Alimentaria en las comunidades. Uno de los proyectos más importantes son las Escuelas de Campo, desarrolladas en las comunidades el Terrero y Mamey a partir del 2004. Para realizar el estudio de evaluación de impacto del proyecto Escuelas de Campo, se evaluaron a las familias que culminaron el proceso Escuelas de Campo, mediante encuestas realizadas en los hogares de cada uno de los beneficiarios y entrevistas a los técnicos que apoyaron la ejecución del proyecto. En el estudio se revelan los principales hallazgos: el proyecto escuelas de campo brindó a los participantes conocimientos de Manejo Integrado de Cultivos y Manejo Integrado de Plagas, comercialización, conservación de suelos y agua; dando como resultado el incremento en el área de producción, diversificación de parcelas de las familias beneficiadas, contribuyendo a la disponibilidad, acceso y mejoramiento del patrón de consumo de alimentos. Un impacto positivo es la diversificación en las parcelas de los beneficiarios; las familias han introducido nuevos rubros, como Hortalizas, Frutas y Tubérculos; un $47.62 \%$ de las familias cultivan los tres rubros básicos (Maíz, Frijol, Sorgo) y han incorporado a sus parcelas Hortalizas, tales como: Tomate, Chiltoma, Repollo, Cebolla; un 14,29\% de las familias beneficiarias cultivan los tres rubros básicos
ABSTRACT
The current reality of the municipality of San José de Cusmapa, Madriz, raises the necessity to perform programs that contribute to solve problems of Food Security of the communities. One of the most important projects is the Field School (escuelas de campo), developed in the communities of El Terrero and Mamey since year 2004. In order to evaluate the impact of the field school project, the families who culminated the process were objet of study. Surveys were done in the homes of each one of the beneficiaries, as well as, interviews for technicians who worked in the implementation of the project. This study reveals that "Field Schools" offered to the participant knowledge in Integrated Crop Management, Integrated Pest Management, commercialization, and water and soil conservation, leading to an increase in the area planted and diversification of production of the beneficiaries. This situation contributed to the availability and access of food, and improved the pattern of food intake. A positive impact is the diversification in the production of the beneficiaries. The families have introduced new crops such as vegetables, fruits and tubers. Forty eight percent of the families grow the three basic crops (corn, bean, sorghum) and have incorporated to their areas vegetables such as tomato, sweet pepper, cabbage and onion. Fourteen percent of the families grow the three basic grains, as well as, vegetables and fruits (avocado, citruses, and mangos) and a $4.8 \%$ have incorporated vegetables, fruits and tubers to their basic grains. Nine per 
un 4,76\% incorporó a sus parcelas Hortalizas, Frutas y Tubérculos, cultivando siempre los tres rubros básicos; el 9,52\% de los beneficiarios cultivan Frutas y Tubérculos más Maíz, Frijol y Sorgo.

Palabras Clave: Seguridad Alimentaria, Impacto, Escuelas de Campo, Disponibilidad, Acceso, Diversificación, Nivel de Vida, Conocimientos. cent of the beneficiaries grow fruits and Tubers, plus corn, common bean and sorghum.

Keywords: Food security, Impact, Practice Schools, Availability, Access, Diversification, Standard of life, Knowledge.

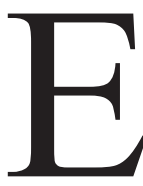
1 presente estudio se realizó entre los meses de Agosto 2008 - Mayo de 2009, en el marco del convenio firmado entre la Universidad Nacional Agraria, de Nicaragua y la Universidad Politécnica de Madrid, España, con el fin de evaluar el impacto en la Seguridad Alimentaria del proyecto Escuelas de $\mathrm{Campo}^{1}$ en las comunidades El Terrero y Mamey, en el período comprendido entre los meses de Agosto 2008 - Mayo 2009.

En Nicaragua, la primera experiencia de Escuelas de Campo fue en el municipio de San Francisco Libre en el 2003. En año 2004 inicia el Proyecto "Desarrollo productivo y Fortalecimiento de las capacidades técnicas y humanas dirigido a pequeñas familias campesinas de las comunidades, el Terrero y el Mamey, mediante la aplicación de la metodología de Escuelas de Campo (ECAs).

En el Municipio de San José de Cusmapa se ha implementado una serie de proyectos enmarcados en salvaguardar la Seguridad Alimentaria de los habitantes de la zona, ya que "San José de Cusmapa, ocupa el octavo puesto de los municipios más pobres del país, con niveles de pobreza de un $83.4 \%$ de su población y un nivel de extrema pobreza del $46.6 \%$, con una tasa de morbilidad infantil entre los más alarmantes del país, con 48 de cada 1,000 nacidos vivos".

Las comunidades El Terrero y Mamey fueron seleccionadas para la implementación del proyecto escuelas de campo en el 2004 por sus características geográficas de difícil acceso, grandes problemas de abastecimiento de agua potable y condiciones de salubridad.

\section{MATERIALES Y MÉTODOS}

Caracterización de la zona de estudio. El municipio de San José de Cusmapa departamento de Madriz, ubicado a $274 \mathrm{~km}$ de Managua y a $34 \mathrm{~km}$ de Somoto, limita al Norte con el municipio de Las Sabanas (Madriz), al Sur con los municipio de San Juan de Limay (Estelí) y

1 El proyecto Escuelas de Campo fue gestionado y ejecutado por el Instituto de Formación Permanente y Universidad Campesina (INSFOP/UNICAM).

San Francisco del Norte (Chinandega.), al Este Con el Municipio de San Juan de Limay (Estelí), al Oeste con el Municipio San Francisco del Norte (Chinandega) y la República de Honduras. El municipio se divide en siete microrregiones, las comunidades el Terrero y el Mamey se encuentran en la sexta microrregión, a una distancia del casco urbano de $8 \mathrm{~km}$ en el caso de la comunidad el Mamey y a $10 \mathrm{~km}$ la comunidad el Terrero. Cada una de las comunidades cuenta con 265 y 240 habitantes respectivamente.

Diseño Metodológico del Estudio. El tipo de estudio es descriptivo cuali-cuantitativo. El constructo del estudio es el Impacto en Seguridad Alimentaria, tomando en cuenta como principales variables la Disponibilidad de alimentos, el Acceso de alimentos, el Consumo de alimentos y el Nivel de vida.

La población del estudio está representada por las 2 comunidades en las que estuvo presente el proyecto: el Terrero y el Mamey, con una participación de 38 productores como alumnos de las ECA's (familias beneficiarias). Además de tomar en cuenta a los principales Ejecutores del proyecto.Los instrumentos utilizados para la realización de estudio fueron: Encuestas dirigidas a la población efectiva del proyecto (26 graduados beneficiarios) y Entrevista dirigida a los técnicos facilitadores de las Escuelas de Campo.

RESULTADOS Y DISCUSIÓN
Disponibilidad de alimentos
Producción interna. En la Tabla 1 se presenta el total
de la superficie cultivada de granos básicos por los
beneficiarios de las escuelas de campo antes y después
del proyecto. Antes de la inserción de los beneficiarios
al proyecto escuelas de campo, la superficie de siembra
destinada para el cultivo de maíz era de 13.52 ha, ahora
se cultivan 19.29 ha, se observa un crecimiento del
$42.68 \%$. El rubro de frijol pasó de 11.77 ha cultivadas a
17.18 ha, creció en un $45.96 \%$, mientras que el cultivo de
sorgo es el que ocupa menos superficie de siembra, sin
embargo, éste no dejó de incrementar en su producción
en un $33.11 \%$, pasando de 7.37 ha, a 9.81 ha cultivadas.


El área total cultivada antes del proyecto era de 32.66 ha, después del proyecto hubo un incremento a 46.28 ha equivalente al $41.70 \%$.

Tabla 1. Total de superficie (ha) cultivada de granos básicos antes y después de la participación de los beneficiarios en las Escuelas de Campo

\begin{tabular}{lrrcc}
\hline Cultivos & Antes & Ahora & $\begin{array}{c}\text { Crecimiento } \\
\text { Absoluto (ha) }\end{array}$ & $\begin{array}{c}\text { Crecimiento } \\
\text { Relativo (\%) }\end{array}$ \\
\hline Maíz & 13.5 & 19.3 & 5.8 & 42.7 \\
Frijol & 11.8 & 17.2 & 5.5 & 46.0 \\
Sorgo & 7.4 & 9.8 & 2.4 & 33.1 \\
\hline Total & 32.7 & 46.3 & 13.6 & 41.7 \\
\hline
\end{tabular}

En la Tabla 2 se observan los rendimientos promedios en la última cosecha de granos básicos y los rendimientos promedios obtenidos antes de haber participado en las sesiones de escuelas de campo. Los rendimientos promedios para el rubro de maíz antes era de $517.71 \mathrm{~kg} \mathrm{ha}^{-1}$, en la actualidad ha aumentado en un $45 \%$, obteniendo $750.67 \mathrm{~kg} \mathrm{ha}^{-1}$. Los rendimientos para el rubro del frijol no pasaban de $673.02 \mathrm{~kg} \mathrm{ha}^{-1}$, ahora obtienen rendimientos de $757.15 \mathrm{~kg} \mathrm{ha}^{-1}$, lo que equivale a un crecimiento del $12.5 \%$. El cultivo de sorgo/millón presenta un incremento del $18.24 \%$, ya que ha pasado de $1100.13 \mathrm{~kg} \mathrm{ha}^{-1}$ a $1300.74 \mathrm{~kg} \mathrm{ha}^{-1}$.

Tabla 2. Rendimientos promedios de la Producción de granos básicos antes y después de la participación en las Escuela de Campo

\begin{tabular}{lcccc}
\hline Cultivos & Antes (kg) & Ahora (kg) & Diferencia (kg) & \% \\
\hline Maíz & 517 & 750.1 & 232.4 & 45.0 \\
Frijol & 673 & 757.1 & 84.1 & 12.5 \\
Sorgo/Millón & 1100 & 1301.0 & 200.6 & 18.2
\end{tabular}

Si comparamos estos datos con los rendimientos de frijol, maíz y sorgo/millón cosechados por las familias beneficiarias después de su participación en las Escuelas de Campo, se obtiene que los rendimientos del cultivo de frijol se igualan a los rendimientos de la media nacional del ciclo 2007/2008, los rendimientos del cultivo de maíz son un $45.8 \%$ inferiores a la media nacional y los rendimientos de sorgo/millón difieren en $5.2 \%$, siendo mayores los rendimientos nacionales por un porcentaje mínimo.

Diversificación de cultivos por las familias beneficiarias. Antes de que fueran participes los beneficiarios de ambas comunidades (Terrero y Mamey), únicamente se dedicaban a las labores agrícolas tradicionales, como era la siembra de Maíz, Frijol y Sorgo/Millón, donde el $57.1 \%$ de las familias beneficiarias sembraban los tres rubros básicos antes mencionados, un $38.1 \%$ cultivaba solamente Maíz y Frijol y un $4.8 \%$ cultivaba únicamente Maíz y Sorgo/Millón.

Con la participación en el proyecto las familias han introducido nuevos rubros, tales como Hortalizas, Frutas y Tubérculos; un $47.6 \%$ de las familias cultivan los tres rubros básicos (Maíz, Frijol, Sorgo) y han incorporado a sus parcelas Hortalizas como Tomate, Chiltoma, Repollo, Cebolla, un $14.29 \%$ de las familias beneficiarias cultivan los tres rubros básicos mas Hortalizas y Frutas (Aguacate, Cítricos, Mangos) y un $4.76 \%$ incorporó a sus parcelas Hortalizas, Frutas y Tubérculos cultivando siempre los tres rubros básicos, el 9.52\% de los beneficiarios cultivan Frutas y Tubérculos mas el Maíz, Frijol y Sorgo.El 38.10\% del total de familias que cultivaba Maíz y Frijol disminuyó a $14.29 \%$ y estas incorporaron en sus parcelas las Hortalizas y las Frutas; el 23.8\% de las familias restante incluyeron en sus parcelas el cultivo de Sorgo/Millón y pasaron a cultivar además de Maíz y Frijol, el cultivo de Sorgo/Millón. El 4.76\% que cultivaban Maíz y Sorgo/ Millón incorporaron las Hortalizas y las Frutas.

Acceso a Alimentos. En la tabla 3 se observan los ingresos económicos promedios mensuales obtenidos por las familias beneficiarias, donde el 57.7\%, correspondiente a la mayoría de las familias beneficiarias, tienen ingresos entre 500 y 1125 córdobas, y una minoría del $7.69 \%$ tienen ingresos entre 2376 a 3000 córdobas mensuales.

Las familias beneficiarias presentan un ingreso mensual promedio de 1266.7 córdobas por familia. Estos ingresos son obtenidos en época de cosecha por la venta de la producción; en época que no hay producción los jefes de familia se ven obligados a emigrar de las comunidades a vender su mano de obra a los cortes de café en países vecinos como Honduras, y algunas mujeres emigran a las ciudades a trabajar como domésticas.

$\mathrm{Si}$ se compara los ingresos de las familias beneficiarias, se obtiene que el $57.7 \%$ de las familias viven en la línea de Pobreza, ya que presentan ingresos mensuales entre 500 y 1125 córdobas, obteniendo ingresos menores a los 2 dólares diarios, el $43.31 \%$ restante presentan ingresos mayores a los 2 dólares por día y ningún beneficiario se encuentra en la línea de extrema pobreza. 
Tabla 3. Ingresos Económicos Promedios mensuales

\begin{tabular}{rcc}
\hline \multicolumn{1}{c}{ Ingresos } & Frecuencia & Porcentaje \\
\hline $500-1125$ & 15 & 57.7 \\
$1126-1750$ & 4 & 15.4 \\
$1751-2375$ & 5 & 19.2 \\
$2376-3000$ & 2 & 7.7 \\
\hline Total & 26 & 100 \\
\hline
\end{tabular}

Precio de los alimentos. La oferta de alimentos en el municipio se basa más en productos básicos (maíz, frijol) y en menor escala las hortalizas, en lo que refiere a cereales estos son destinados para el autoconsumo. El precio de los granos básicos en las comunidades el Terrero y Mamey ha sufrido fluctuaciones en sus precios como se observa en la figura 1.

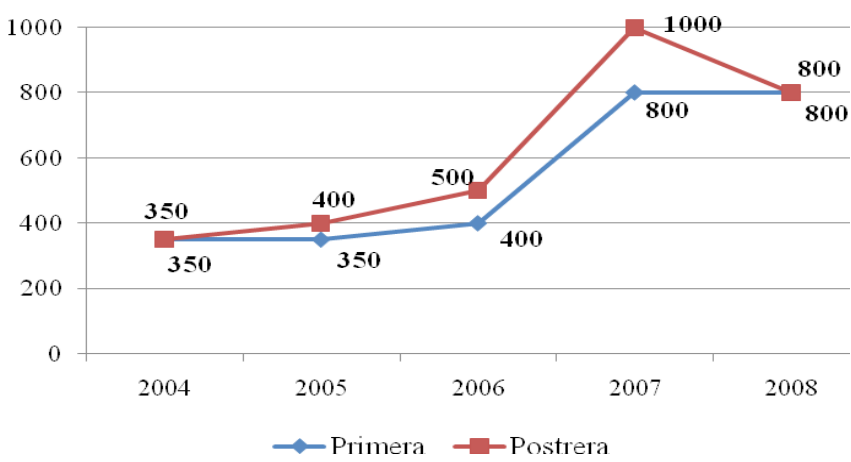

Figura 1. Variación de precios del frijol en las comunidades en estudio.

Los precios de frijol en las comunidades el Terrero y Mamey, han tenido una variación desde el año 2004 al 2008 que va de 350 córdobas en el año 2004 a 800 córdobas en la cosecha del ciclo agrícola 2008.

El precio de los alimentos afecta el suministro y la demanda de los mismos. Los precios bajos dan menos ingresos a los agricultores por sus productos y si caen demasiado no podrán producir o vender. Sin embargo, los precios bajos representan un aumento en la capacidad de compra del consumidor.

La reducción del precio de un alimento básico como el maíz equivale a aumentar el ingreso de todos los que compran esos alimentos. Por otro lado, si se aumenta el precio de los productos los ingresos del consumidor disminuyen.

Uno de los instrumentos más usados en América Latina para medir accesibilidad a alimentos es la canasta básica de alimentos o canasta familiar. Al realizar la comparación de los Ingresos Promedios Mensuales de cada familia beneficiaria con la Canasta Básica Urbana y la Canasta Básica Rural, se determinó la Capacidad Adquisitiva, siendo esta apenas de un $14.6 \%$ para cubrir el costo de la CBU lo que corresponde a menos de la cuarta parte de dicho costo y con un $61 \%$ de capacidad para cubrir el costo de la CBR, lo que significa que si ellos gastaran todos sus ingresos mensuales en alimentos les haría falta C\$ 825.33 para completar su canasta.

Consumo de alimentos. En el caso de las familias beneficiarias con el proyecto escuelas de campo, consumen lo que producen; antes del proyecto no variaban lo hábitos alimenticios debido a labores agrícolas tradicionales (siembra de frijol y maíz). Luego de la participación de las familias en las Escuelas de Campo, su situación mejoró, gracias a la adaptabilidad de conocimientos en técnicas agrícolas, manejo de suelos, cosechas de agua, lo cual les permitió variar las labores agrícolas a cultivos no tradicionales y así mejorar sus hábitos de consumo.

Patrón de consumo de alimentos. El grupo de alimentos consumidos con mayor frecuencia por las familias beneficiarias, son frijol, maíz, huevo, café y azúcar que conforman la dieta diaria del $100 \%$ de las familias.

Alimentos tales como: arroz, sorgo/millón (consumido en atol, tortillas), guineo verde y hortalizas (cebolla, chiltoma y tomate) son consumidos con frecuencia de 2 a 4 veces a la semana, los cuales complementan un poco la dieta de las familias. Dieta que es muy rica en proteínas gracias al consumo de alimentos como frijol, huevo y maíz, siendo el azúcar el producto que les brinda mayor cantidad de energía.

En época de cosecha los alimentos que son consumidos con frecuencia de 3 a 5 veces por semana, están; Cítricos (limón, naranja), Aguacate y Ayote.

Los alimentos complementarios son consumidos con menor frecuencia, ya que estos alimentos tienen que ser adquiridos en las pulperías y por lo general los consumen cuando en el hogar hay algún tipo de ingreso económico. En este grupo de alimentos el aceite es el único que es utilizado por el $100 \%$ de las familias beneficiarias, ya que este es utilizado en la preparación de alimentos tales como: el pollo, arroz y huevos que es preparado de diferentes maneras (frito entero, en torta, revuelto, etc.)

En época de cosecha, y cuando los beneficiarios obtienen más ingresos el 100\% de las familias añaden a su dieta alimentos como: carne de res, espagueti, sopas magui y refrescos preparados, aprovechando al máximo los ingresos obtenidos por la venta de la producción. 


\section{Nivel de vida}

Vivienda. En cuanto a la tenencia de la vivienda, el $100 \%$ de las viviendas son propias y construidas con paredes de adobe. El tipo de piso es de tierra en un $95.24 \%$ de las familias y un $4.76 \%$ es de embaldosado. El techo del $38.10 \%$ de las viviendas es de zinc, un $47.62 \%$ de las viviendas tiene techo de teja y el $14.29 \%$ son de zinc y teja. En síntesis, el $9.52 \%$ de las viviendas en que habitan las familias beneficiarias se encuentran en buen estado, el $66.67 \%$ en regular estado y el $23.81 \%$ en mal estado.

Educación. En la figura 2 se muestra el nivel de escolaridad de las familias beneficiarias, en la que se observa que el $56 \%$ de los beneficiarios tiene un nivel de escolaridad de primaria (entre segundo y quinto grado), un $19 \%$ secundaria media, el $10 \%$ pertenece a una población menor de 4 años por lo que no posee ningún grado de educación, un $8 \%$ es analfabeta; en comparación con los datos presentados en la caracterización del municipio por PRESANCA en el 2007 con 17\% de analfabetismo; se observa que existe un menor grado de analfabetismo en las familias beneficiarias, un 3\% de la población ha sido alfabetizada por el programa Yo $\mathrm{Si}$ Puedo, y un $4 \%$ cursa el preescolar.

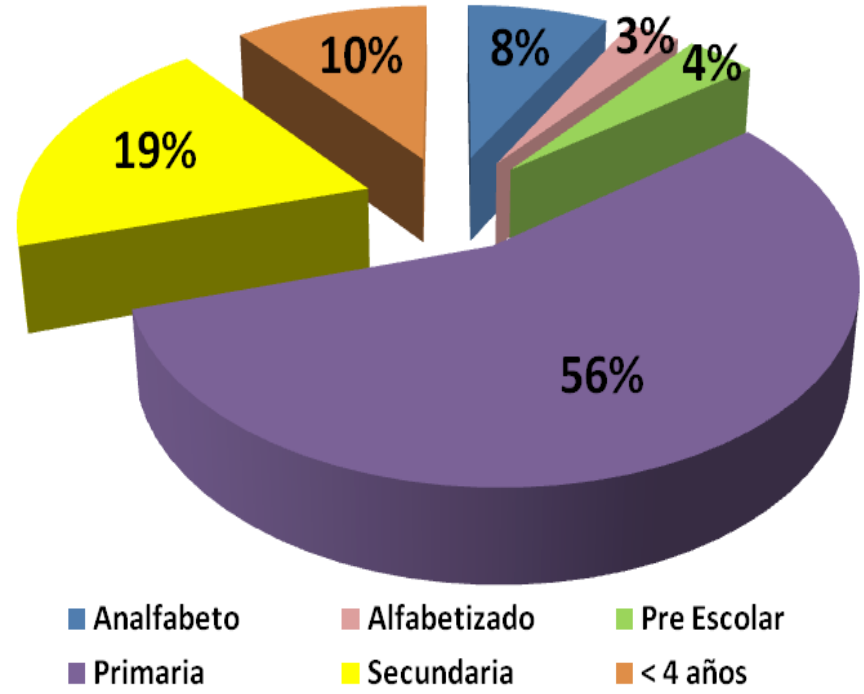

Figura 2. Nivel de escolaridad de las Familias beneficiarias.

Ocupación de la población. En La distribución ocupacional de las familias beneficiarias, el $46 \%$ es estudiante y pertenece a la población en edades entre 6 y 20 años, el $23 \%$ ama de casa, $21 \%$ se dedica a las labores agrícolas y un $10 \%$ corresponde a los menores de 4 años de edad (sin ocupación).
Distribución de la población económicamente activa e inactiva. En la figura 3 se observa la distribución de la población beneficiaria por rango de edades y sexo, esta pirámide presenta un comportamiento donde en la actualidad, la mano de obra está compuesta por jóvenes y mujeres, se observa que el mayor peso está hasta los 24 años, esto es significativamente positivo siempre y cuando se logre una estrategia de incorporar en actividades productivas a estos jóvenes. Según la caracterización del municipio de San José de Cusmapa elaborada por PRESANCA en el 2007, el 34\% de la PEA del municipio se dedica a la realización de actividades informales, empleos temporales en la agricultura, existiendo tendencia a emigrar hacia otras zonas del país en busca de trabajo durante las épocas de cosecha.

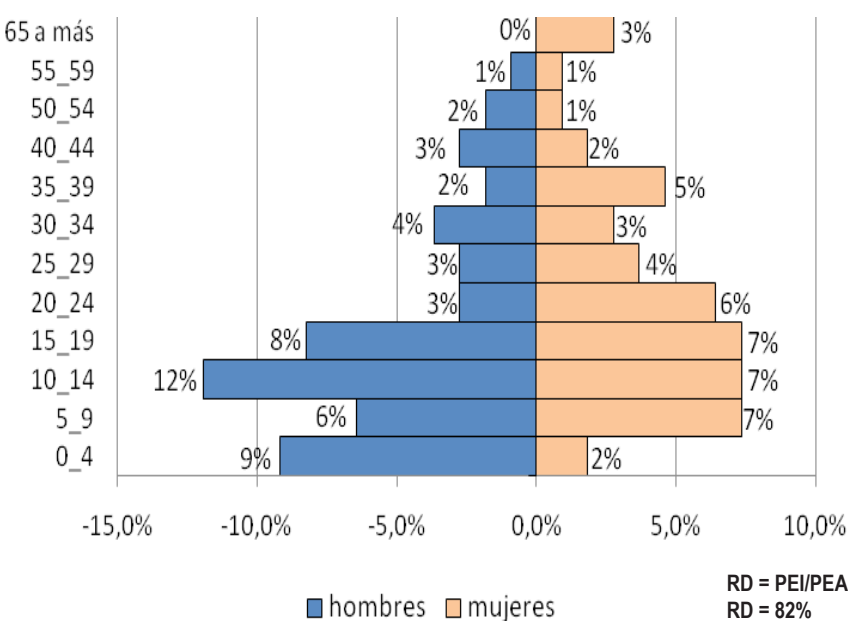

Figura 3. Pirámide poblacional según el rango de edad y sexo de la población beneficiaria.

En base a los rangos de edades por sexo presentados en la figura 3, se obtiene la Razón de Dependencia de la población, el $55 \%$ de la población se encuentra dentro de la Población Económicamente Activa (PEA) presentando 10 puntos porcentuales más que la PEA nacional, ya que según el censo nacional de población y vivienda del año 2005 el $45.2 \%$ de la población pertenece a la PEA.

La razón de dependencia calculada en las comunidades equivale al $82 \%$, lo que significa que de cada 100 personas económicamente productiva, 82 dependen económicamente de estas. Con relación al promedio del número de miembros que componen las familias beneficiarias, el cual es de cinco personas por familia, se obtiene que cuatro de estas son económicamente dependientes de una persona económicamente productiva, lo cual hace aun más difícil la situación alimentaria. 


\section{Cobertura a servicio de salud y saneamiento}

Agua potable. El $86 \%$ de las familias beneficiarias cuentan con servicio de agua potable, que llega hasta sus hogares a través de mangueras conectadas a un sistema de mini acueducto por gravedad, asegurando que el agua es de buena calidad, aprovechando este servicio para el establecimiento de sistemas de riego por aspersión para la siembra de cultivos en los meses de verano, el 14\% no cuenta con un servicio de agua en su hogares.

Servicio de letrina. El $100 \%$ de las familias beneficiarias un 76,19\% tienen acceso a letrinas en sus hogares, de las cuales el $23,81 \%$ se encuentran en buen estado, un $47,62 \%$ en estado regular y apenas el $4,76 \%$ están en mal estado. Un $23,81 \%$ de las familias no cuentan con acceso a letrinas, por lo que realizan sus necesidades fisiológicas al aire libre, lo que representa un peligro de contaminación en fuentes de agua y propagación de enfermedades.

Servicio de salud. Para asistir a las comunidades el Terrero y Mamey, existe un centro de salud ubicado en el Mamey, en este únicamente permanece una enfermera y un médico que llega una vez por semana. Cuando se trata de una enfermedad de mayor gravedad, los habitantes de las comunidades deben recorrer dos horas a pie o en bestias para llegar al centro de salud del casco urbano.

Enfermedades más comunes. Como se observa en la figura 5, no se presentan enfermedades graves, las enfermedades más comunes y con mayor porcentaje son Tos y Resfriados con un 29\% y $38 \%$ respectivamente. Se realizó la pregunta a las familias beneficiarias sobre los hábitos de higiene y el 100\% respondió que tratan de cumplir con la higiene en los hogares.

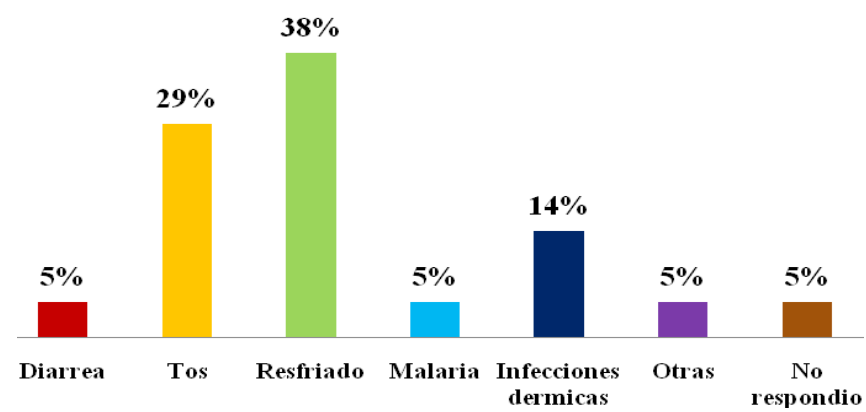

Figura 5. Enfermedades de mayor frecuencia en la población beneficiada.

\section{CONCLUSIONES}

La implementación de la metodología de Escuelas de Campo para Agricultores en las comunidades el
Terrero y Mamey, ha tenido un impacto positivo en el mejoramiento de la Seguridad Alimentaria en las familias beneficiarias.

El aporte conocimientos adquiridos a través de la metodología Escuelas de Campo, ha mejorado la disponibilidad de alimentos, ya que las familias beneficiarias han incrementado su área de producción principalmente en granos básicos.

Uno de los principales resultados esperados por la implementación de la metodología de escuelas de campo, es que se logró que los beneficiarios por medio del aprendizaje sobre el manejo integrado de cultivos como hortalizas, tubérculos, frutales, se apropiaran de estos conocimientos llevándolos a la práctica en sus parcelas, pasando de una producción tradicional a una diversificada, de esta manera contribuyen a mejorar la disponibilidad de alimentos en el hogar.

El incremento significativo en los rendimientos de los cultivos (granos básicos) ha sido producto del esfuerzo de los beneficiarios por la aplicación de los conocimientos adquiridos sobre Manejo Integrado de Plagas y Manejo Integrado de Cultivos, conservación de suelo y agua y creación de bancos de semilla.

Un logro importante fue la inserción de la mujer en el proceso de producción y comercialización de los productos obtenidos, principalmente en la transformación de alimentos.

Con la producción diversificada en granos básicos, frutas y hortalizas, los beneficiarios tienen un patrón de consumo alimentario más variado, ya que de la producción que obtienen el $50 \%$ es para el autoconsumo y el $50 \%$ restante es para la venta, de la cual obtienen ingresos que contribuyen a la compra de alimentos complementarios.

Aunque el patrón de consumo de alimentos está determinado por el tamaño y composición de la familia, la dieta entre los miembros de las familias, no varía, ya que según la población encuestada todos comen por igual, es decir que existe una equidad en la distribución del tipo de alimentos consumidos.

Los alimentos consumidos con mayor frecuencia por las familias beneficiarias. Alimentos como frijol, maíz, huevo, café y azúcar conforman la dieta diaria del $100 \%$ de las familias.

El nivel de vida de los beneficiarios ha mejorado de forma significativa en comparación a los años anteriores y a las estadísticas municipales, ya que con la adquisición de nuevos conocimientos se crea una nueva visión de la problemática de Seguridad Alimentaria en los hogares. 


\section{REFERENCIAS BIBLIOGRÁFICAS}

Ardón, M. (18 de Septiembre de 2003). Aprendiendo con las ECAS. LEISA Revista de Agro ecología, Volumen (19), p. 29-34

FAO (Organización de las Naciones Unidas para la Agricultura y la Alimentación). 2002. Seguridad Alimentaria y Nutricional. Consultado el día 26 de mayo del 2008 de la World Wide Web: http://www.rlc.fao.org/prior/ segalim/prodalim/prodveg/bpa.

INIDE (2005). Censos Nacionales 2005, Censo de Población, Vivienda y Hogar. Managua, NI. 220 p.

PRESANCA (2007). Caracterización del Municipio de San José Cusmapa. Programa Regional de Seguridad Alimentaria.

PROCOSAN (2006). Análisis de la situación nutricional del municipio de San José de Cusmapa. Programa Comunitario de Salud y Nutrición.

Ruiz A.; Marín Y. (2005). Revisando el Agro Nicaragüense. Nitlapan-UCA (Ed.), Managua, NI. 134 p.

Trueba, I; Angulo, A. (2006). El fin del hambre en el 2025. Aedos S.A (Ed.), Las ECAs y análisis de sus potencialidades en los procesos de Desarrollo Rural UPM, España. 345 p. 